\section{Prevalência de dispareunia na gravidez e fatores associados}

\section{The prevalence of dyspareunia in pregnancy and associated factors}

\begin{abstract}
Objectives: to establish the prevalence of dyspareunia in the third gestational trimester and associated factors.

Methods: 202 puerperal women were evaluated. Data was collected using a socio-demographic questionnaire; the ICIQ-Short Form questionnaire; an intestinal constipation questionnaire; and, questions from the FSFI questionnaire to evaluate dyspareunia. The data were analyzed using the chi-squared, MannWhitney $U$ and Wilcoxon tests, $p<0.05$.

Results: the prevalence of dyspareunia in the third gestational trimester was 48.5\% $(n=98)$, compared with $15.3 \%(n=31)$ before gestation. The factors associated with dyspareunia in this period were: constipation in the third trimester of gestation $(p=0.05)$; urinary incontinence in the third trimester of gestation $(p<0.001)$ and dyspareunia prior to gestation $(p=0.01)$. The mean age of women with dyspareunia was significantly higher than that of those without dyspareunia $(p=0.01)$.

Conclusions: the prevalence of dyspareunia is high during gestation and is associated with alterations in the functions of the pelvic saddle, such as the presence of urinary incontinenceand constipation, and prior dyspareunia.
\end{abstract}

Key words Dyspareunia, Pregnancy, Sexual behaviour, Pelvic floor
Fabiana Flores Sperandio 1

Cinara Sacomori 2

Isabela dos Passos Porto 3

Fernando Luiz Cardoso 4

1-4 Centro de Ciências da Saúde e do Esporte. Universidade do Estado de Santa Catarina. Rua Pascoal Simone, 358. Coqueiros. Florianópolis, SC, Brasil. CEP: 88.080-350. E-mail: fabi@intercorp.com.br

\section{Resumo}

Objetivos:verificar a prevalência de dispareunia no terceiro trimestre gestacional e fatores associados.

Métodos: foram avaliadas 202 puérperas. Para a coleta de dados foram utilizados um questionário sociodemográfico; questionário ICIQ-Short Form; questionário de constipação intestinal e, para a avaliação da dispareunia foram utilizadas as questões do questionário FSFI. Os dados foram analisados com os testes qui quadrado, $U$ de Mann Whitney e teste de Wilcoxon, $p<0,05$.

Resultados: a prevalência de dispareunia no $3^{\circ}$ trimestre gestacional foi de 48,5\% $(n=98)$, enquanto antes da gestação era de $15,3 \%(n=31)$. Os fatores associados à dispareunia nesse periodo foram: constipação no terceiro trimestre da gestação ( $p=0,05)$; incontinência urinária no terceiro trimestre $d a$ gestação $(p<0,001)$ e dispareunia antes $d a$ gestação $(p=0,01)$. A média de idade das mulheres com dispareunia foi significativamente maior que as sem dispareunia $(p=0,01)$.

Conclusões: a prevalência da dispareunia é alta no periodo gestacional e esta associada a alterações das funções do assoalho pélvico, como presença de incontinência urinária e constipação, além da presença prévia de dispareunia.

Palavras-chave Dispareunia, Gravidez, Comportamento sexual, Diafragma da pelve 


\section{Introdução}

A gravidez é um período em que as mudanças na mecânica corporal são mais acentuadas e ocorrem concomitantemente graças as adaptações relacionadas ao processo de gestar. ${ }^{1}$ Os músculos do assoalho pélvico são tracionados para baixo em função do aumento do peso uterino e acabam por comprometer a contratilidade dessa musculatura. ${ }^{1}$ Além disso, tendem a aparecer veias varicosas na região da vulva ou no interior da vagina, as quais podem ocasionar desconforto ou dor durante o ato sexual, a que se chama dispareunia. ${ }^{2}$

A dispareunia constitui-se em uma disfunção sexual gerada por alterações físicas ou psicológicas ${ }^{3}$ e pode afetar a qualidade de vida das mulheres. ${ }^{4}$ Esta disfunção é caracterizada como "queixa de dor persistente ou recorrente ou desconforto associado com tentativa ou a completa penetração vaginal".5 Ocorre em cerca de $15 \%$ das mulheres entre 30 e 50 anos de idade, 6 varia entre 23 e $41 \%$ no terceiro trimestre gestacional7,8 e de 30 a $60 \%$ entre as mulheres no pós-parto, ${ }^{9}$ período em que coexistem sentimentos e/ou atitudes negativas em relação ao sexo.

Segundo a literatura, as disfunções sexuais durante a gestação estão associadas a fatores psicológicos, 10 físicos, 11 socioculturais/religiosos 12,13 e relacionais. 14 Além da dispareunia, outras fases do ciclo de resposta sexual (desejo, excitação, lubrificação, orgasmo e satisfação) estão alteradas principalmente no terceiro trimestre gestacional.10,13 Diversos estudos indicam que a queixa de dispareunia parece aumentar com o avançar da gestação 15,16 afetando até $80 \%$ das mulheres no último trimestre. 17

No estudo realizado no Brasil por Lima et al., 18 foi identificado que a dispareunia é uma disfunção sexual presente em primigestas e que a prevalência de algum tipo de disfunção sexual, antes da gravidez, é menor do que a apresentada durante a gestação. Quando analisada por trimestre gestacional, os autores encontraram um acréscimo da disfunção sexual conforme o aumento da idade gestacional. A prevalência da ocorrência de qualquer um dos problemas na função sexual (falta de desejo, diminuição da lubrificação, insatisfação e presença de dor durante as relações sexuais) passou de $54,1 \%$ no primeiro trimestre, para $66,3 \%$ no terceiro trimestre de gestação. Outro estudo brasileiro identificou que a dispareunia teve um aumento durante a gestação, sendo que no primeiro trimestre a prevalência foi de $22,5 \%$, no segundo de $33,8 \%$ e, no terceiro chegou a $44,3 \% .19$
No Brasil são poucos os estudos que visam identificar a dispareunia no período gestacional, bem como os fatores a ela associados. Assim, o objetivo deste estudo é verificar a prevalência de dispareunia no terceiro trimestre gestacional e os fatores associados.

\section{Métodos}

Esta pesquisa se caracteriza como um estudo de corte transversal cujas medidas relacionadas ao período antes de gestação e terceiro trimestre gestacional foram retrospectivas. Participaram do estudo 202 mulheres que se encontravam no puerpério imediato em um hospital e maternidade de referência na cidade de Florianópolis, entre o período de agosto de 2011 e março de 2012. O estudo teve aprovação no Comitê de Ética em Pesquisa (CAE 0010.0.233.269-11). O critério de inclusão na pesquisa abrangeu mulheres submetidas ao procedimento de parto normal ou cesáreo e que estivessem sexualmente ativas no terceiro trimestre de gestação. Os critérios de exclusão foram: apresentar incontinência urinária de origem neurológica, ter histórico de câncer no trato geniturinário, apresentar dificuldades de compreensão das questões/analfabetismo, ser cega, possuir diagnóstico de doenças neurológicas, ter menos de 18 anos e ser usuária de drogas.

Para a coleta de dados foi utilizada uma ficha com dados sociodemográficos contendo história clínica, número de gestações, ganho de peso na gestação, prática de atividade física (antes e durante a gestação) e autorrelato de problemas associados (diabetes, asma, bronquite, depressão e tabagismo). Tais dados foram obtidos na consulta ao prontuário hospitalar e/ou por meio do autorrelato das pacientes. Para a avaliação das perdas urinárias, foi utilizado o questionário ICIQ - Short Form, traduzido e validado para português por Tamanini et $a l .20$ em 2004. Esse questionário avalia a frequência e a quantidade de perda urinária, a situação desta perda de urina e a interferência nas atividades de vida diária. Foram consideradas incontinentes as mulheres que referiram qualquer episódio de perda nesse período.

Também foi utilizado um questionário estruturado com critérios relacionados à constipação intestinal, considerando-se os dois períodos: prégestação e terceiro trimestre gestacional. A constipação foi avaliada de acordo com os critérios Roma $^{21}$ : sensação de bloqueio anal durante a defecação; necessidade de uso da facilitação digital para defecação; sensação de evacuação incompleta; passagem de fezes duras; ocorrência de menos de 
três evacuações por semana; uso regular de laxantes ou utilização de enemas. A constipação foi caracterizada entre as pacientes que apresentaram três ou mais desses critérios.

Para avaliação da dispareunia, aplicaram-se as três últimas perguntas do questionário Female Sexual Function Index (FSFI), o qual foi desenvolvido nos Estados Unidos 22 e validado para a língua portuguesa por Latorre et al. ${ }^{23}$ e Pacagnella et $a l .{ }^{24}$ As perguntas referem-se à frequência e a intensidade de dispareunia durante e após o coito nos períodos pré-gestacional e III trimestre gestacional. $\mathrm{O}$ questionário foi aplicado no pós-parto imediato. $\mathrm{O}$ escore de dispareunia foi obtido mediante a soma das três questões e multiplicadas por 0,4 ; sendo que quanto maior o escore, menor a dor associada ao intercurso sexual. As perguntas foram: "Qual a frequência da dispareunia?" / "Qual a intensidade da dispareunia durante a penetração?" / "Qual a intensidade da dispareunia após a penetração?”.22 O estudo avaliou a dispareunia inespecífica, sem realizar um aprofundamento em suas causas.

Para a referida pesquisa, o procedimento de coleta dos dados consistiu em explicar os objetivos e os benefícios da pesquisa à puérpera e solicitar a assinatura do termo de consentimento livre e esclarecido. A coleta de dados ocorreu no próprio leito hospitalar.

Os dados foram analisados mediante o uso do programa Statistical Package for the Social Sciences (SPSS) versão 20.0. A forma de apresentação consistiu em estatística descritiva (frequências absolutas e relativas, média, mediana, desvio padrão) e inferencial (teste Qui-quadrado, teste U de Mann Whitney e teste de Wilcoxon). Foi adotado $p<0,05$.

\section{Resultados}

Em geral, as participantes do estudo caracterizavamse por viverem com seus parceiros $(90 \%)$, possuírem escolaridade de nível médio $(58,7 \%)$ e etnia caucasiana $(86,6 \%)$ como descrito na Tabela 1 .

A média de idade foi de 26,5 anos $(s d=6,25)$. Com relação às características obstétricas foi identificado que a média de ganho de peso na gestação foi de $13,59(\mathrm{sd}=6)$ e a mediana do número de gestações foi 2 (Tabela 2 ).

Como indicam as Tabelas 1 e 3, a prevalência de dispareunia no terceiro trimestre gestacional foi $48,5 \%(n=98)$, sendo que a frequência de dispareunia reportada foi de quase sempre ou sempre $(35,7 \%)$, a maioria das vezes $(18,4 \%)$, as vezes $(28,6 \%)$ e algumas vezes $(17,3 \%)$. Com relação à intensidade da dispareunia a maioria $(58,2 \%, n=57)$ relatou como sendo moderada. Antes da gestação a prevalência da dispareunia era de $(16,1 \%, n=39)$ sendo que a maioria $(67,7 \%, \mathrm{n}=21)$ relatou ter às vezes dor durante o ato sexual.

Observou-se associação entre a dispareunia no terceiro trimestre de gestação e a constipação no terceiro trimestre $(p=0,05)$, incontinência urinária no terceiro trimestre $(p<0,001)$ e dispareunia antes da gestação $(p=0,01)$; de modo que as mulheres que apresentavam dispareunia no terceiro trimestre de gestação tiveram maiores proporções de dispareunia antes da gestação $(23,5 \%)$ e maiores proporções de constipação no terceiro trimestre $(32,7 \%)$ comparado as que não tinham queixas de dispareunia no $3^{\circ}$ trimestre. A média de idade das mulheres com dispareunia foi significativamente maior que as sem dispareunia (Tabela 2).

Ao comparar a dispareunia antes da gestação e no terceiro trimestre gestacional, foi observado que a frequência e a intensidade da dispareunia aumentaram no terceiro trimestre (Tabela 3).

\section{Discussão}

No presente estudo evidenciamos que a dispareunia apresentou maior prevalência durante o terceiro trimestre gestacional comparada à antes da gestação. Identificamos que os fatores associados a esta disfunção foram a idade, a presença de incontinência urinária e a constipação no terceiro trimestre da gestação. A dispareunia é um problema que afeta grande número de mulheres, inclusive no período gestacional.4,8 Constitui-se em uma disfunção sexual caracterizada por dor antes, durante ou após o intercurso sexual.2,4,8 Esta disfunção sexual na gestação é experimentada por cerca de 22 a $50 \%$ das mulheres, apesar de que $12 \%$ destas, em média, vivenciarem o desconforto no estado pré-gravídico. 25

Tem sido amplamente sustentado que, o fato de mulheres sentirem dor e desconforto durante a penetração, leva a uma diminuição da motivação para a atividade sexual e, consequentemente, diminui a sua frequência em ocasiões subsequentes. 25 Nosso estudo não incluiu as mulheres que não realizaram atividade sexual no terceiro trimestre gestacional, devido à limitação do questionário de dispareunia. Este instrumento não nos permite comparar o período pré-gravídico com o gestacional no que se refere à prática da atividade sexual, uma vez que a não realização levaria a diminuição do escore do questionário no terceiro trimestre gestacional. Assim, não poderíamos inferir que a não realização tenha sido decorrente da dispareunia ou de outros fatores. 26 
Tabela 1

Caracterização sociodemográfica e de saúde das participantes do estudo com e sem dispareunia, no terceiro trimestre gestacional.

\begin{tabular}{|c|c|c|c|c|c|c|c|}
\hline \multirow[t]{2}{*}{ Características } & \multicolumn{2}{|c|}{ Todas $(\mathrm{N}=202)$} & \multicolumn{2}{|c|}{$\begin{array}{l}\text { Com dispareunia } \\
(n=98)\end{array}$} & \multicolumn{2}{|c|}{$\begin{array}{l}\text { Sem dispareunia } \\
\qquad(n=98)\end{array}$} & \multirow[t]{2}{*}{$X^{2}(p)$} \\
\hline & $\mathrm{n}$ & $\%$ & $\mathrm{n}$ & $\%$ & $\mathrm{n}$ & $\%$ & \\
\hline Escolaridade & & & & & & & NS \\
\hline Fundamental & 64 & 31,8 & 33 & 33,6 & 31 & 30,1 & \\
\hline Médio & 118 & 58,7 & 57 & 58,2 & 61 & 59,2 & \\
\hline Superior & 19 & 7,8 & 8 & 8,2 & 11 & 10,7 & \\
\hline Estado marital & & & & & & & $x^{2}=4,1(p=0,04)$ \\
\hline Com companheiro & 181 & 90,0 & 91 & 92,9 & 90 & 86,5 & \\
\hline Sem companheiro & 20 & 10,0 & 6 & 6,2 & 14 & 13,5 & \\
\hline Etnia & & & & & & & NS \\
\hline Branca & 175 & 86,6 & 84 & 85,7 & 91 & 89,2 & \\
\hline Negra/mulata & 25 & 12,4 & 14 & 14,3 & 11 & 10,8 & \\
\hline \multicolumn{8}{|l|}{ Problemas associados } \\
\hline Diabetes & 13 & 5,3 & 5 & 5,1 & 3 & 2,9 & NS \\
\hline Bronquite crônica & 18 & 7,4 & 10 & 10,2 & 6 & 5,8 & NS \\
\hline Asma & 7 & 10,3 & 4 & 4,1 & 1 & 1,0 & NS \\
\hline Hipertensão & 29 & 11,9 & 15 & 15,3 & 9 & 8,7 & NS \\
\hline Depressão & 14 & 5,8 & 8 & 8,2 & 3 & 2,9 & NS \\
\hline Tabagismo & 58 & 23,9 & 26 & 26,5 & 23 & 22,1 & NS \\
\hline Constipação AG & 76 & 31,4 & 37 & 37,8 & 31 & 29,8 & NS \\
\hline Constipação $3^{\circ}$ trimestre & 63 & 26,0 & 32 & 32,7 & 25 & 24,0 & $x^{2}=3,74(p=0,05)$ \\
\hline Atividade física AG & 107 & 44,2 & 44 & 44,9 & 47 & 45,2 & NS \\
\hline Atividade física $3^{\circ}$ trimestre & 78 & 32,2 & 29 & 29,6 & 38 & 36,5 & NS \\
\hline $\begin{array}{l}\text { Incontinência urinária } \\
3^{\circ} \text { trimestre }\end{array}$ & 144 & 59,5 & 69 & 70,4 & 55 & 52,9 & $x^{2}=8,12(p=0,004)$ \\
\hline Dispareunia AG & 39 & 16,1 & 23 & 23,5 & 8 & 7,7 & $x^{2}=6,58(p=0,01)$ \\
\hline
\end{tabular}

AG=antes da gestação.

Tabela 2

Comparação entre as mulheres com e sem dispareunia quanto a idade, número de gestações e ganho de peso na gestação.

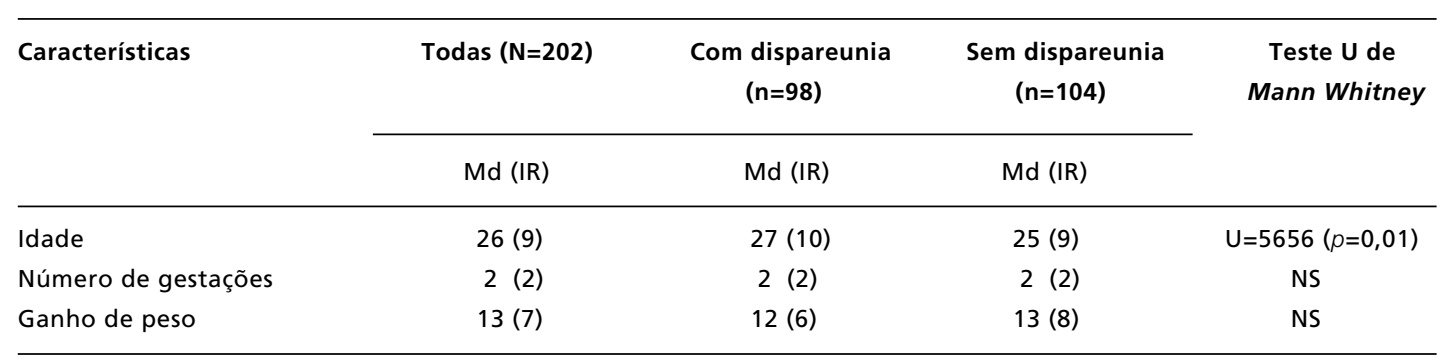

$M d=$ mediana; $I R=$ intervalo interquartil; NS=não significativo para $p<0,05$. 
Comparação da dispareunia antes da gestação e no $3^{\circ}$ trimestre gestacional.

\begin{tabular}{|c|c|c|c|c|c|c|c|}
\hline & \multicolumn{2}{|c|}{$\bar{x}$} & \multicolumn{2}{|c|}{ Md } & \multicolumn{2}{|c|}{ dp } & \multirow{2}{*}{$\begin{array}{l}\text { Teste de } \\
\text { Wilcoxon } p^{*}\end{array}$} \\
\hline & AG & III & AG & III & AG & III & \\
\hline $\begin{array}{l}\text { Frequência da dispareunia } \\
\text { durante a penetração* }\end{array}$ & 4,6 & 3,0 & 5 & 3,5 & 0,9 & 1,9 & $<0,001$ \\
\hline $\begin{array}{l}\text { Frequência da dispareunia } \\
\text { após a penetração* }\end{array}$ & 4,7 & 3,6 & 5 & 5 & 0,8 & 1,9 & $<0,001$ \\
\hline Intensidade da dispareunia* & 4,6 & 3,3 & 5 & 4 & 0,8 & 1,7 & $<0,001$ \\
\hline Escore da dispareunia** & 5,63 & 4,0 & 6 & 5 & 0,9 & 2,1 & $<0,001$ \\
\hline
\end{tabular}

$\overline{\mathrm{X}}=$ média, $\mathrm{dp}=$ desvio padrão; $\mathrm{md}=$ mediana; $\mathrm{AG}=$ Antes da gestação; III=terceiro trimestre; * Escala de resposta das perguntas ( $1=$ muito baixo ou nulo, 2 =baixo, $3=$ moderado, $4=a l$ ta, $5=$ alto); **Corresponde ao somatório das três questões anteriores multiplicado por 0,4 .

A prevalência maior de dispareunia no terceiro trimestre de gravidez, comparado ao período prégestacional, pode ser explicada pelo fato de que neste período as mulheres se encontram em maior vulnerabilidade, apresentando maior irritabilidade decorrente de contrações uterinas provocadas pelo orgasmo, desconforto nas posições sexuais, percepção subjetiva de falta de atratividade física ou, ainda, graças ao aborrecimento pela percepção de diminuição de satisfação por parte do companheiro. 26

Nosso estudo encontrou uma prevalência de dispareunia de $48,5 \%$ no terceiro trimestre gestacional corroborando com as características supracitadas. Este resultado é similar ao encontrado em estudos sobre a temática. $8,9,11,14$ No estudo realizado por Sobhgol et al.,27 incluindo grávidas e não grávidas, eles identificaram que a dispareunia esteve associada ao período da gestação, e a prevalência foi maior na população de gestantes.

Da mesma forma Hart et al.,28 observaram que a dispareunia era comum na gestação e aumentava a prevalência em 50\% no terceiro trimestre. Gokyildiz e Beji17 observaram que a dispareunia esteve mais presente no terceiro trimestre de gestação, aumentando progressivamente e que, a frequência do intercurso sexual diminuía, concomitantemente, com a evolução da gestação. Entretanto, no estudo de Leite et al. 16 a dispareunia no terceiro trimestre diminuiu e os autores justificaram tal fato à prevalência de mulheres que não realizaram a atividade sexual nesse período.

Esta pesquisa encontrou associação entre dispareunia e constipação, o que já havia sido descrito por Sobhgol et al.27 Nesse caso, a constipação crônica pode prejudicar a saúde e a qualidade de vida, pois facilita o aparecimento de lesões como os prolapsos de diferentes órgãos pélvicos e a incontinência urinária de esforço.21 Sabe-se que as mudanças anatomo-fisiológicas impostas pela gravidez têm repercussões variadas nos diferentes sistemas. Para tal, o congestionamento pélvico e, em particular, a vasocongestão do canal vaginal são responsáveis em parte pela dispareunia. ${ }^{1}$

Além disso, foi relatado que a força do assoalho pélvico esteve associada à dispareunia, em que mulheres que apresentavam menor força eram aquelas que referiam dor durante o ato sexual.27 Esta informação mantém relação com a associação entre a dispareunia e a incontinência urinária presente no estudo em questão. Mulheres com fraqueza do assoalho pélvico tendem a apresentar sintomas de perdas urinárias devido à incapacidade de contenção da urina em situações de esforço ou urgência miccional.

Nesse estudo, a média de idade das mulheres que apresentavam a dispareunia foi maior que as que não relatavam este sintoma. No entanto, Laumann et al., ${ }^{6}$ ao analisar 1749 mulheres não grávidas, encontraram que com o aumento da idade a dispareunia diminui, exceto para aquelas com problemas de lubrificação. Sendo assim, a lubrificação pode, igualmente, influenciar os resultados face às alterações neuro-fisiológicas típicas da gestação.

Nosso estudo não encontrou associação entre a escolaridade e a dispareunia, porém outros estudos apresentam esta associação. Mulheres com melhores níveis educacionais apresentam menor nível de 
dispareunia quando comparado com mulheres com escolaridade inferior. ${ }^{27}$ Isso, supostamente, porque pessoas com melhores níveis educacionais apresentam melhores condições de orientações sobre hábitos saudáveis de vida, como a prática de atividade física, controle do tabagismo, menores números de gestações e partos. ${ }^{26}$ Acreditamos que essa contradição com a literatura possa ser devido as características da amostra em questão, onde a maioria dos nascimentos são realizados pelo Sistema Único de Saúde (SUS). Sendo assim, há fortes indícios de que o poder aquisitivo diferenciado influencie na condição sócio econômico e no estilo de vida, uma vez que apenas $7 \%$ da amostra apresentou nível superior.

Por fim, a prevalência da dispareunia esteve mais elevada no terceiro trimestre comparado ao período pré-gestacional. Podemos identificar, igualmente, que os fatores associados à dispareunia neste período foram o fato destas apresentarem sintomas de constipação e incontinência urinária. Verificamos que a intensidade da dispareunia aumenta no decorrer da gestação bem como a frequência do sintoma.

Como limitações citamos o não controle longitudinal destas mulheres no período gravídico-puerperal e a amostra ter sido coletada em uma maternidade pública o que distancia os dados quanto aos fatores sócio-culturais e demográficos de países em situação econômica mais privilegiada. Diante deste cenário, seria prudente acompanhar e comparar os resultados pré-gestacionais e gestacionais em serviços públicos e privados além do uso de instru-

\section{Referências}

1. Stephenson R, O'connor L. Fisioterapia aplicada à ginecologia e obstetrícia. 2 ed. Barueri: Manole; 2004.

2. Raina R, Pahlajani G, Khan S, Gupta S, Agarwal A, Zippe CD. Female sexual dysfunction: classification, pathophysiology, and management. Fertil Steril. 2007; 88 (5): 127384.

3. Handa VL. Sexual function and childbirth. Semin Perinatol. 2006; 30 (5): 253-6.

4. Davis K, Devinder K, Stuart S. Pelvic floor dysfunction: the need for a multidisciplinary team approach. J Pelvic Surg. 2002; 9 (1): 23-36.

5. Haylen B, Ridder D, Freeman RM, Swift SE, Berghmans B, Lee J, Monga A, Petri E, Rizk D, Sand P, Schaer G. An International Urogynecological Association (IUGA)/International Continence Society (ICS) joint report on the terminology for female pelvic floor dysfunction. Int Urogynecol J. 2010; 21 (5): 26.

6. Laumann EO, Paik A, Rosen RC. Sexual dysfunction in the mentos específicos para o monitoramento dos aspectos psicológicos e inflamatórios/infecciosos.

Neste estudo não foram consideradas as possíveis causas da dispareunia, como infecções, inflamações, traumatismos, em função de que grande parte dos prontuários apresentavam informações incompletas. Igualmente, muitas das puerpéras faziam pré-natal em unidades de saúde e chegavam à maternidade somente no momento do parto. Para tal, estas informações foram omitidas na presente pesquisa com vistas ao controle dos viéses.

Em complemento, como agentes de saúde e pesquisadores entendemos que uma ação interdisciplinar potencializará parte destas queixas, por vezes subnotificadas na assistência clínica cotidiana. $\mathrm{O}$ problema da incontinência urinária e os desconfortos de constipação podem ser minimizados, a curto e longo prazo, com orientações de distintos profissionais, o que poderá prevenir doenças no puerpério remoto. Lamentavelmente, a aderência das gestantes em programas de reabilitação (fisioterapia) ainda carece de incentivo, o que pode ser, em parte, responsável pelos índices de dispareunia em centros de assistência à maternidade, parto e puerpério.

Com base nesse estudo, sugerimos novas pesquisas, e que estas controlem o comportamento da dispareunia ao longo do $1^{\circ}, 2^{\circ}$ e $3^{\circ}$ trimestres gestacionais ou, ainda, com outros grupos populacionais e com distintas situações socioeconômicas e culturais. Também aconselhamos outros estudos com desenhos randomizados e longitudinais, com vistas a avaliarem o efeito de um tratamento preventivo e/ou reabilitador da dispareunia ao longo da gestação.
United States: prevalence and predictors. JAMA. 1999; 281: 537-44.

7. Ferreira DQ, Nakamura MU, Souza E, Neto CM, Ribeiro MC, Santana TG, Abdo CH. Função sexual e qualidade de vida em gestantes de baixo risco. Rev Bras Ginecol Obstet. 2012; 34 (9): 409-13.

8. Tennfjord MK, Hilde G, Stær-Jensen J, Ellström Engh M, $\mathrm{B} ø \mathrm{~K}$. Dyspareunia and pelvic floor muscle function before and during pregnancy and after childbirth. International urogynecology journal [Internet]. 2014 Sep [cited 2014 Nov 16]; 25 (9): 1227-35. Available from: http://www.ncbi.nlm.nih.gov/pubmed/24687365

9. Connolly A, Thorp J, Pahel L. Effects of pregnancy and childbirth on postpartum sexual function: a longitudinal prospective study. Int Urogynecol J Pelvic Floor Dysfunct. 2005; 16: 263-7.

10. Pauleta JR, Pereira NM, Graca LM. Sexuality during pregnancy. J Sex Med. 2010; 7 (1): 136-42. 
11. Kennedy C, Turcea AM, Bradley CS. Prevalence of vulvar and vaginal symptoms during pregnancy and the puerperium. Int J Gynecol Obstet. [Internet]. International Federation of Gynecology and Obstetrics; 2009; 105 (3): 236-9. Available from: http://dx.doi.org/10.1016/j.ijgo. 2009.01 .024

12. Fok WY, Chan LY, Yuen PM. Sexual behavior and activity in Chinese pregnant women. Acta Obstet Gynecol Scand. 2005; 84 (10): 934-8.

13. Shojaa M, Jouybari L, Sanagoo A. The sexual activity during pregnancy among a group of Iranian women. Arch Gynecol Obstet. 2009; 279 (3): 353-6.

14. Bello FA, Olayemi O, Aimakhu CO, Adekunle AO. Effect of pregnancy and childbirth on sexuality of women in ibadan, Nigeria. ISRN Obst Gynecol. [Internet]. 2011 Jan [cited 2014 Nov 11]; 856586. Available from: http://www.pubmedcentral.nih.gov/articlerender.fcgi?artid= $3101881 \&$ tool $=$ pmcentrez\&rendertype $=$ abstract

15. Erol B, Sanli O, Korkmaz D, Seyhan A, Akman T, Kadioglu A. A cross-sectional study of female sexual function and dysfunction during pregnancy. J Sex Med. 2007; 4 (5): 1381-7.

16. Leite AP, Campos AA, Dias AR, Amed AM, De Souza E, Camano L. Prevalence of sexual dysfunction during pregnancy. Rev Assoc Med Bras. 2009; 55 (5): 563-8.

17. Gokyildiz S, Beji KN. The effects of pregnancy on sexual life. J Sex Marital Ther. 2005; 31: 201-15.

18. Lima AC, Dotto LM, Mamede M. Prevalência de disfunção sexual em primigestas, no Município de Rio Branco, Acre, Brasil. Cad Saúde Pública. 2013; 29 (8): 1544-54.

19. Sacomori C, Cardoso, Fernando L. Wittkopf PG, Latorre GFS. Função sexual feminina na gestação. Fisioter Bras. 2012; 13 (6): 458-62.

20. Tamanini JTN, Damdros M, D'ancora CAL, Palma PCRN, Rodrigues NJ. Validação para o português do "International Consultation on Incontinence Questionnaire - Short Form" (ICIQ-SF). Rev Saúde Pública. 2004; 38 (3): 438-44.

Recebido em 4 de agosto de 2015

Versão final apresentada em 18 de dezembro de 2015

Aprovado em 11 de janeiro de 2016
21. Amselem C, Puigdollers A, Azpiroz F, Sala C, Videla S, Fernández-Fraga, X. Whorwell $\mathrm{P}$, Malagelada JR. Constipation: a potential cause of pelvic floor damage? Neurogastroenterol Motil. 2010; 22: 150-e48.

22. Rosen R, Brown C, Heiman J, Leiblum S, Meston C, Shabsigh R, Ferguson D, D'Agostino R. The Famele sexual function Index (FSFI): A multidimensional self-report instrument for the assessment og female sexual function. J Sex Marital Ther. 2011; 26 (2): 191-208.

23. Latorre GFS, Bilck PA, Cardoso FL, Sperandio FF. Validade e confiabilidade de uma versão on-line do Female Sexual Function Index por teste e reteste. Rev Bras Ginecol Obstet. 2013; 35: 469-74.

24. Pacagnella RC, Martinez EZ, Vieira EM. Validade de construto de uma versão em português do Female Sexual Function Index. Cad Saúde Pública. 2009; 25 (11): 233344.

25. Sydow K Von. Sexuality during pregnancy and after childbirth : a metacontent analysis of 59 studies. J Psychosomatic Res. 1999; 47 (1): 27-49.

26. Silva AI, Figueiredo B. Sexualidade na gravidez e após o parto. Psiquiatr Clín. 2005; 25 (3): 253-64.

27. Sobhgol SS, Alizadeli Charndabee SM. Rate and related factors of dyspareunia in reproductive age women: a crosssectional study. Int J Impot Res. 2007; 19 (1): 88-94.

28. Hart J, Cohen E, Gingold A. Sexual behavior in pregnancy: a study of 219 women. J Sex Educ Ther. 1991; 17 (2): 8690 . 